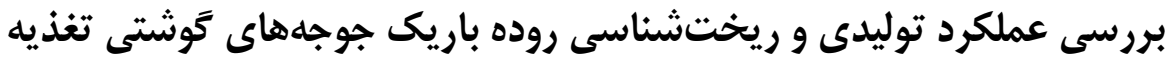

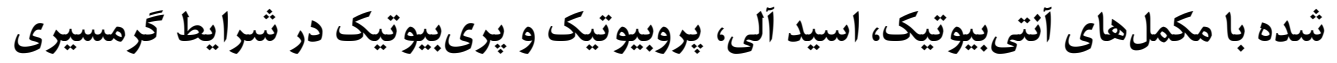

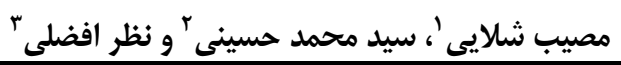

1- كارشناس ارشد، دانشخاه بيرجند، (نويسنده مسوول: mosayeb_shalaey@yahoo.com)

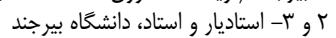

تاريخ دريافت: Tr/N/T

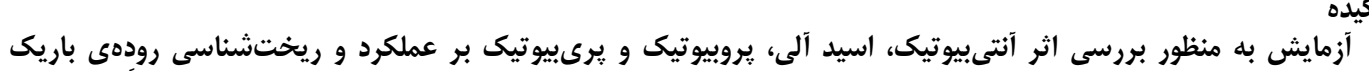

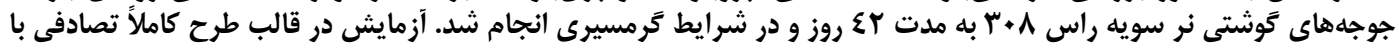

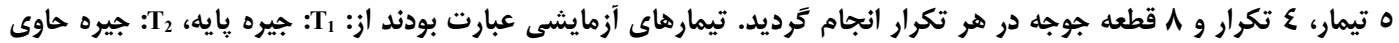

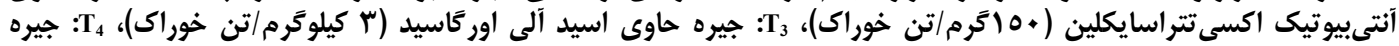

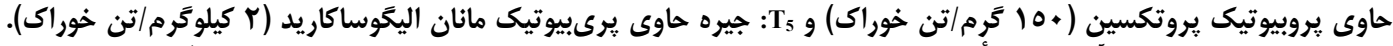

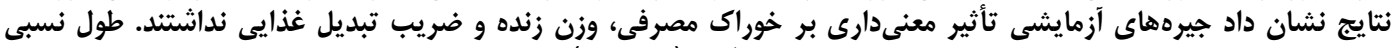

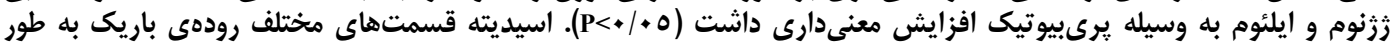

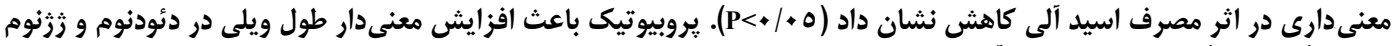

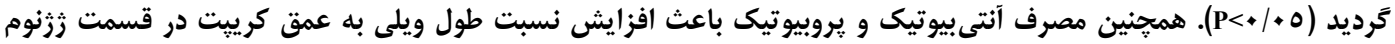

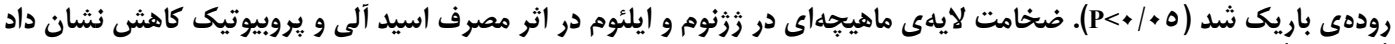

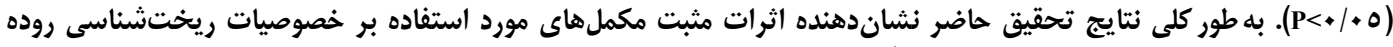

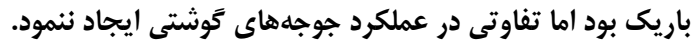

وازههاى كليدى: جوجه توشتى، عملكرد، ريختشناسى روده باريك، مكملهاى غذايى

نظر شكل و اندازه به طور قابل توجهى در هر بخش روار روده

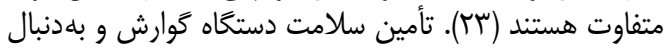

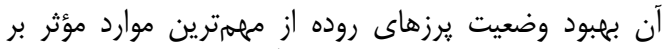

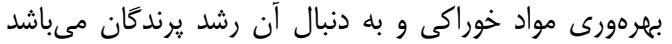

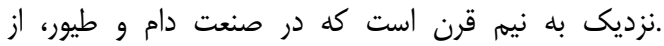

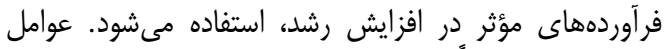

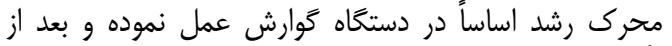

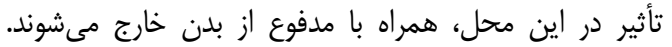

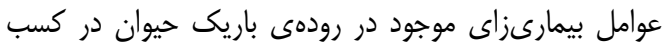

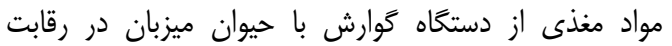

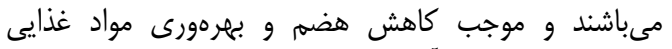

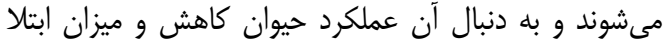

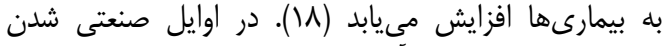

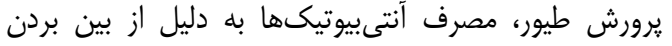

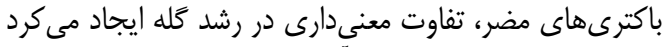

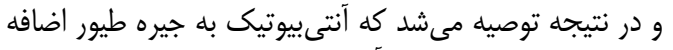

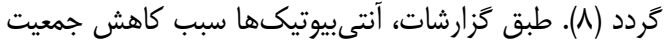

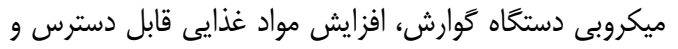

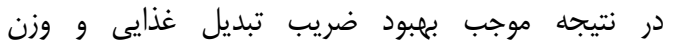

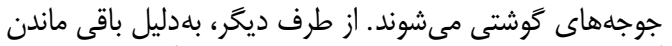

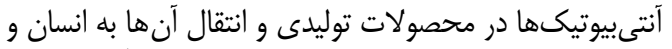

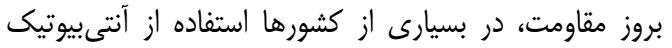

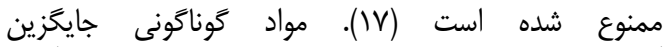

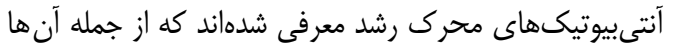

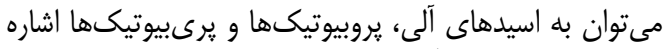

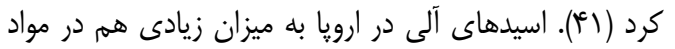

مقدمه

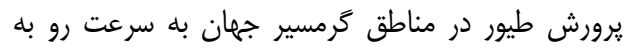
افزايش است. قسمتهاى كثيرى از قارههاى آسيا، آفريقا و و

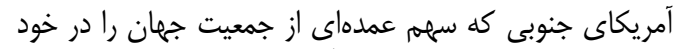

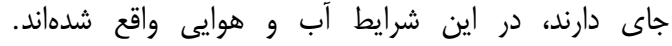

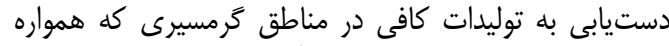

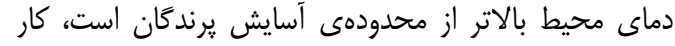

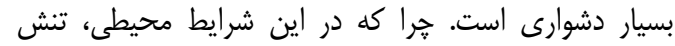

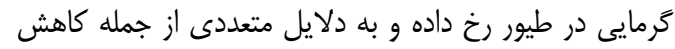

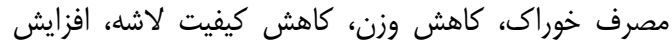

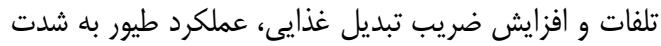

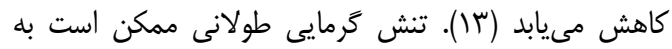

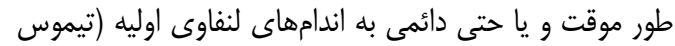

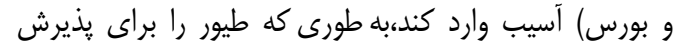

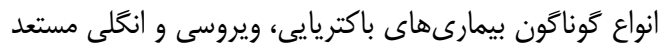

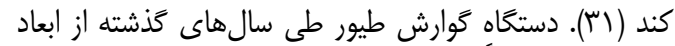

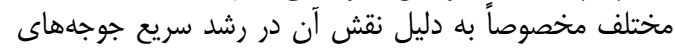

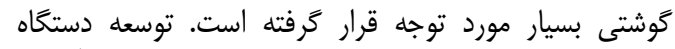

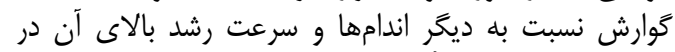

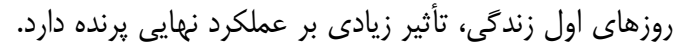

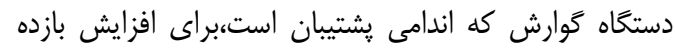

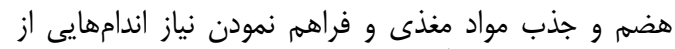

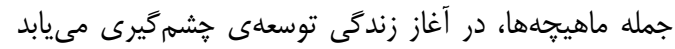

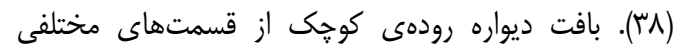

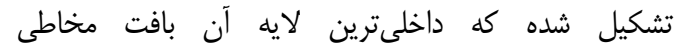

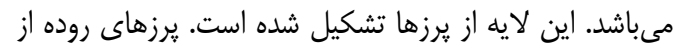




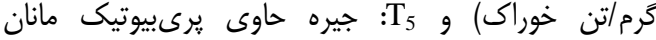

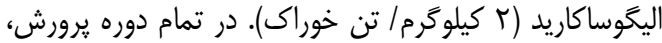

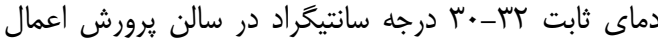

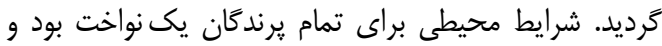

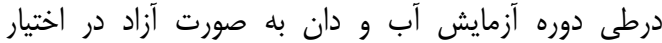

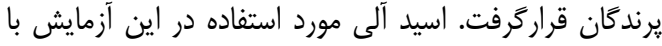

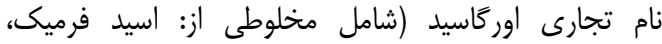

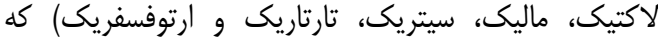

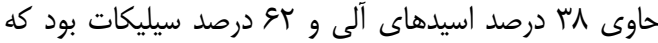

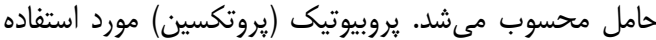

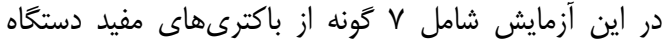

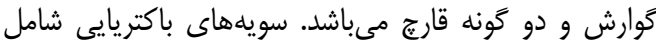

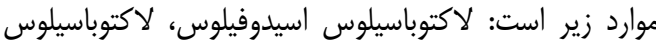

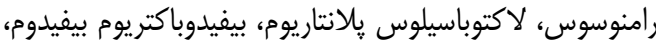

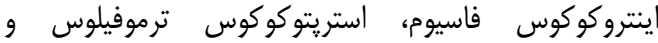

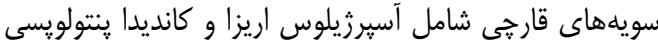

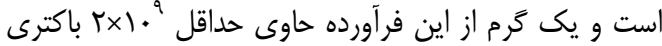

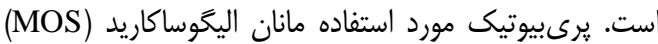

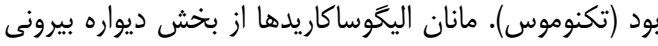

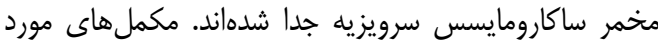

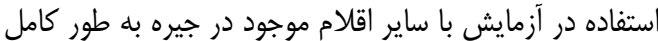

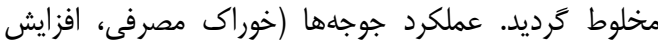

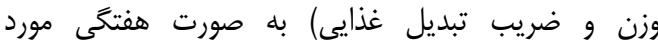

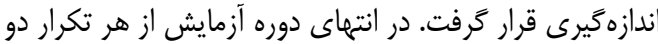

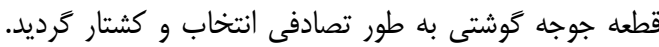

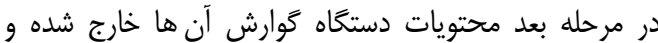

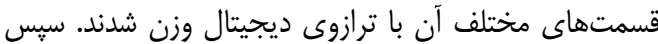

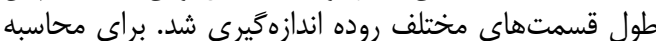
pH

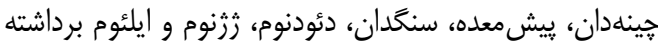

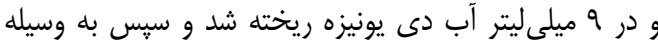

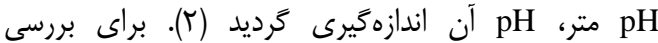

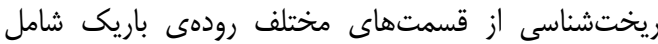

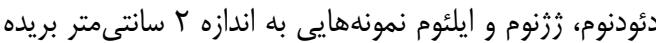

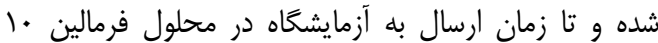

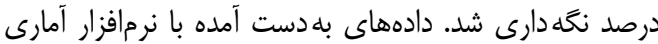

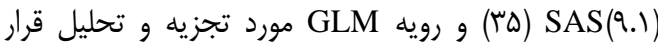

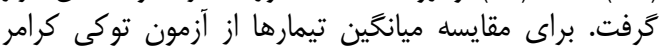
استفاده شد. مدل آمارى طرح به صورت زير مى باشيد: $\mathrm{Y}_{\mathrm{ij}}=\mu+\mathrm{T}_{\mathrm{i}}+\mathrm{E}_{\mathrm{ij}}$

$$
\begin{aligned}
& \text { مقدار هر يك از مشاهدات =Y مئ } \\
& \text { ب ميانغين جمعيت } \\
& \text { ا ثر جيره غذايى } \\
& \text { Eij = اثر خطاى آزمايش }
\end{aligned}
$$

خام غذايى و هم در خوراك آماده، به منظور ممانعت از رشد ماند

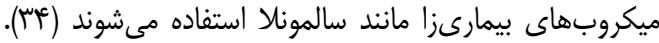

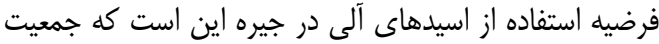

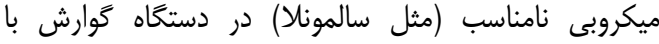

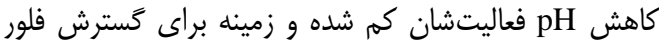

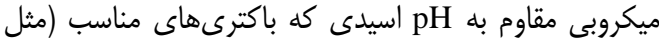

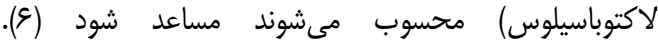

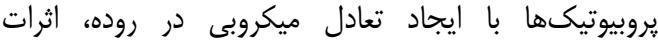

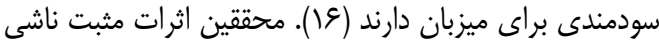

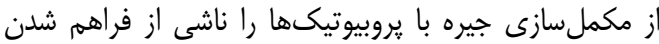

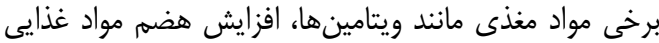

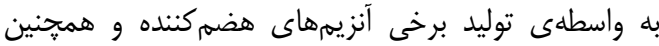

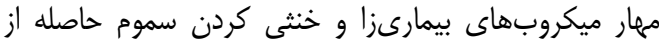

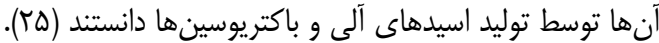

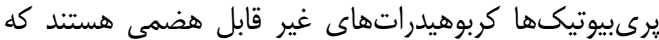

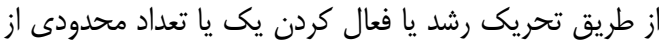

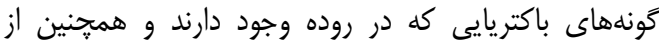

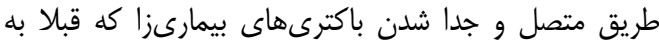

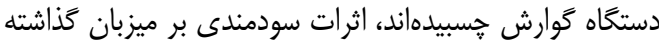

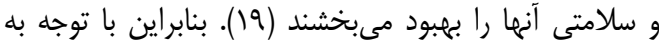

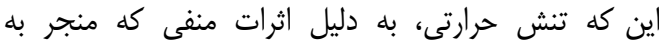

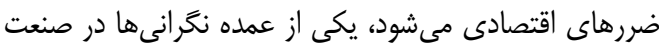

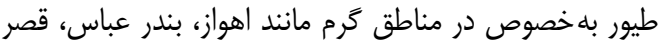

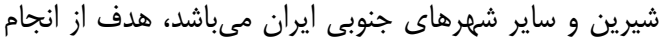

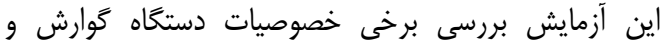

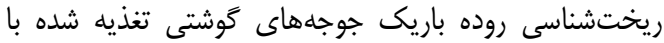

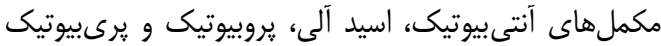
تحت شرايط اقليمى كرمسيرى بودي آني

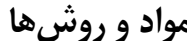

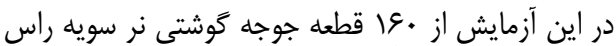

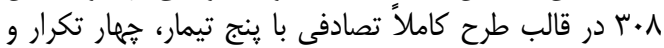

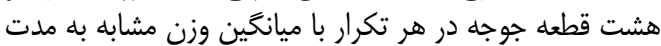

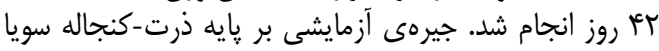

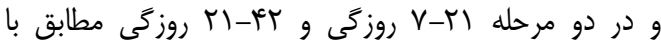

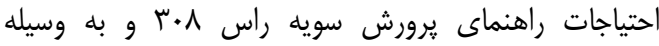

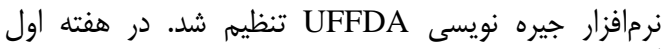

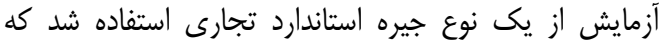

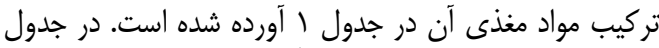

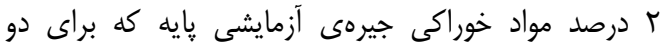

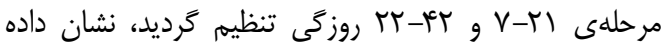

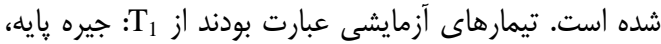

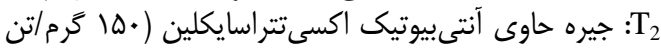

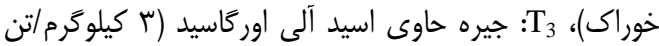

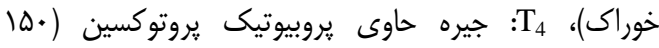


Table 1. Diet composition of the first week

جدول ا- تركيب مواد مغذى جيره هفته اول

\begin{tabular}{|c|c|}
\hline مقدار تأمين شده & 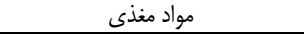 \\
\hline$r \Lambda \Delta \cdot-r q . \cdot$ & انرزى قابل سوخت و ساز (Kcal/kg) \\
\hline rI-Mt & 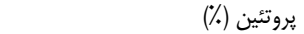 \\
\hline $1 / \cdot$ & كلسيم (\%) \\
\hline$\cdot / 4 \Delta_{-} / \Delta$ & فسفر (\%) \\
\hline$\cdot / \Delta$ & 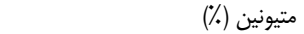 \\
\hline $1 / \pi$ & لايزين (٪) \\
\hline$\cdot / 1$ & متيونين + سيستئين (٪) \\
\hline$\cdot / 19$ & سديم (\%) \\
\hline
\end{tabular}

جدول r- اجزاى تشكيل دهنده (بر حسب درصد) و تركيب مواد مغذى جيره آزمايشى پايه

Table 2. Components and nutrient composition of the basal diet

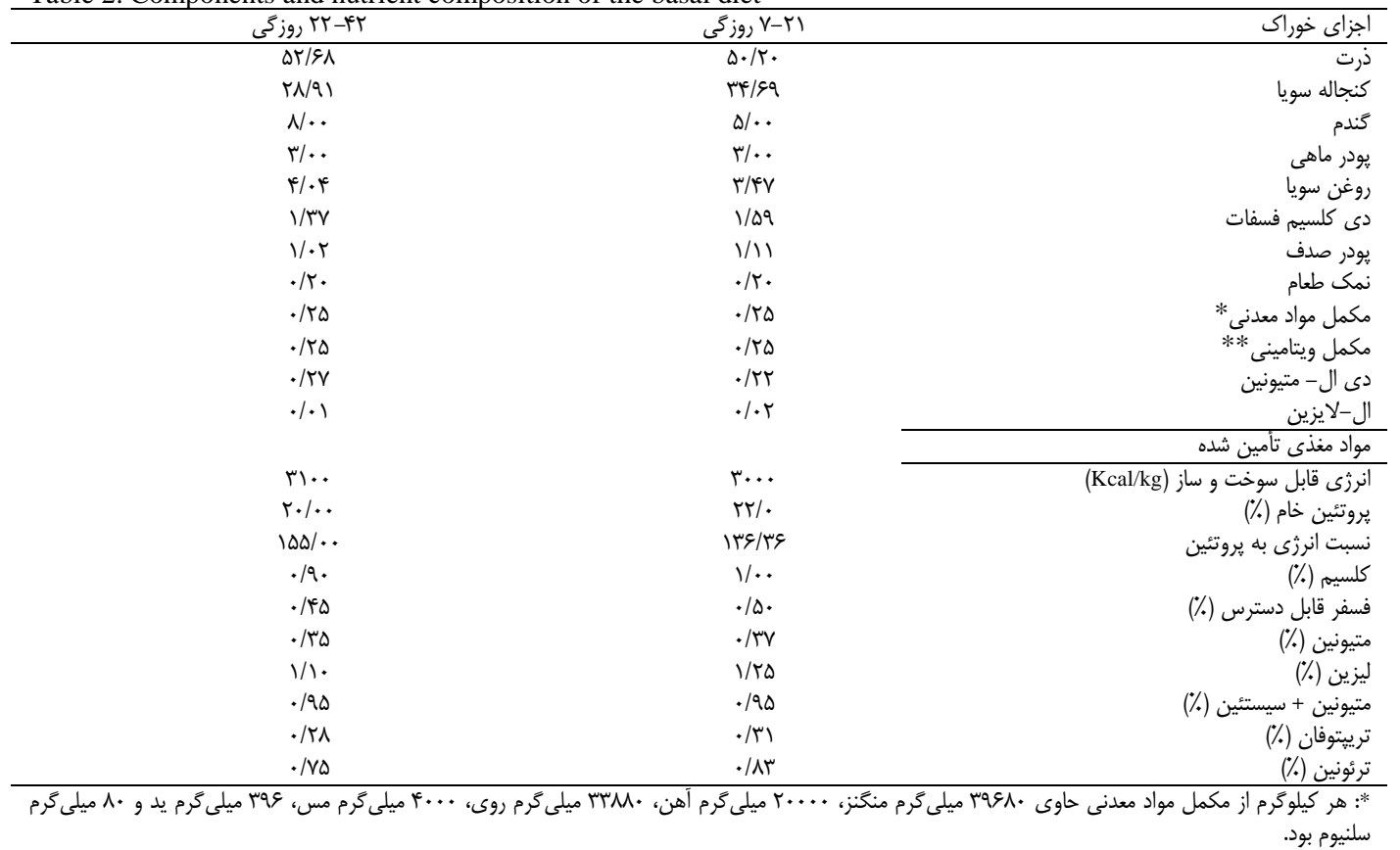

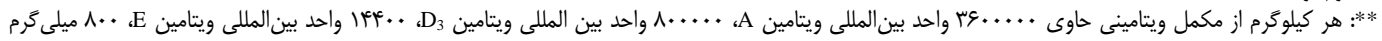

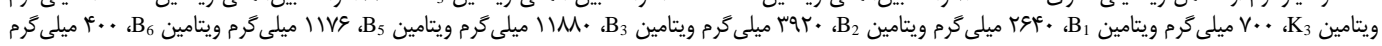

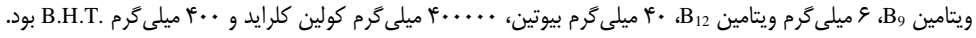

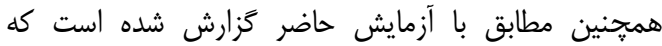

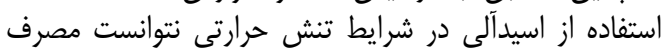

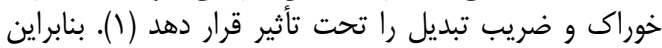

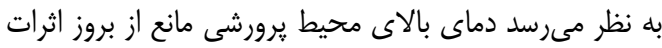

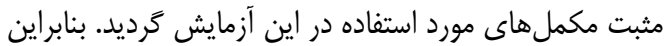

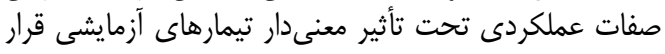
نخرفت.

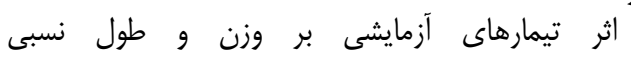

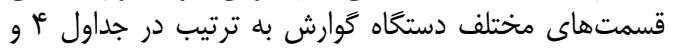

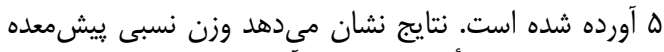

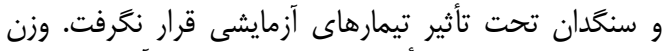

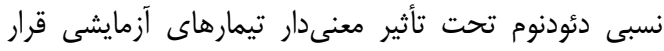

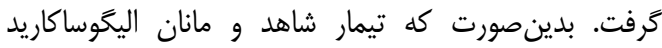

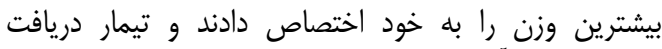
كنندهى اسيد آلى كمترين ميزان را راشت داشت و اين اختلاف دادن به

نتايج و بحث

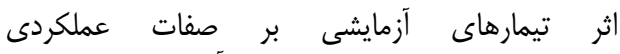
جوجههاى گَشتى در دورههاى مختلف آزمايش درائ در جدول

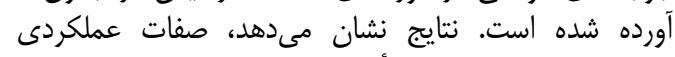

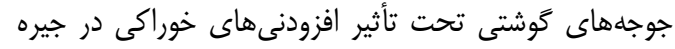

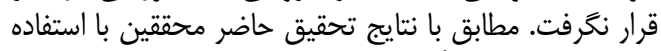

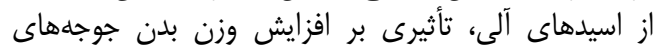

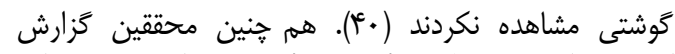

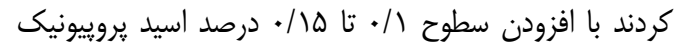

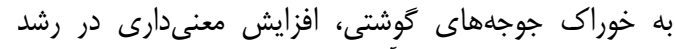

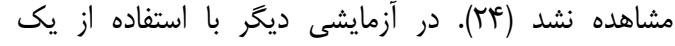

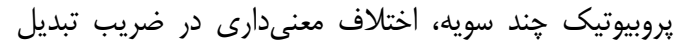

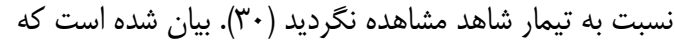

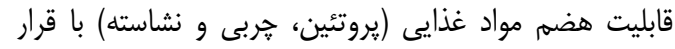

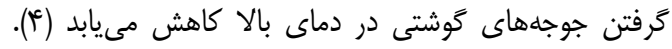


دربارهى طول نسبى قسمتهاى مختلف دستخاه توارش

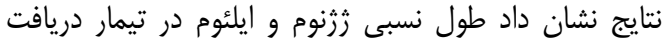

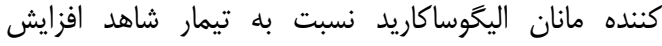

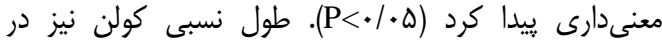

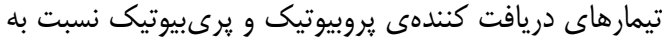

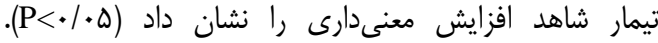

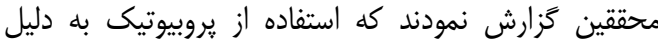

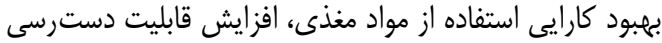

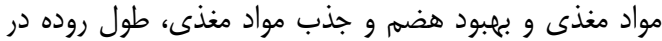

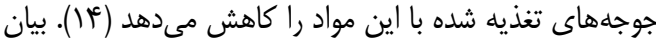

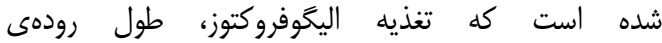

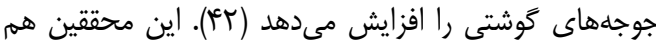

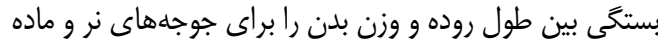

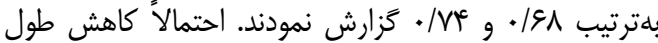

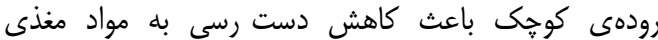

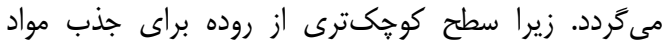

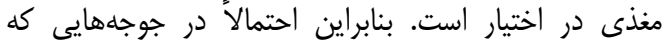

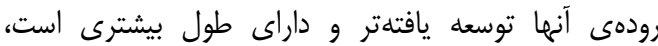

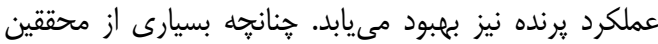

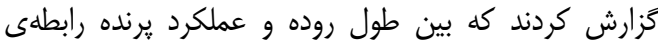

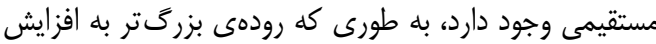

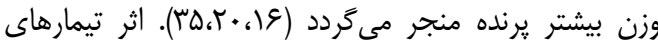

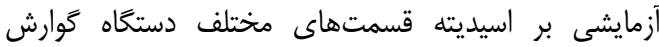
جوجههاى گَوشتى در جدول 9 آورده شده است.

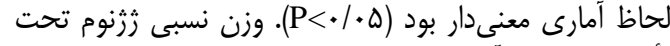

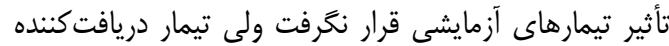

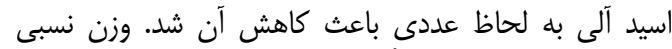

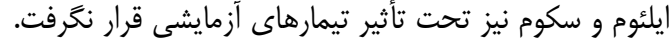

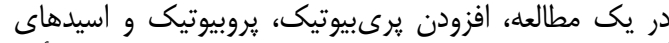

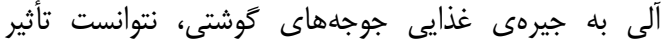

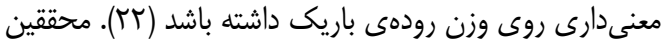

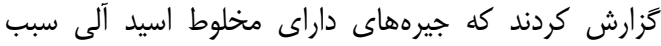

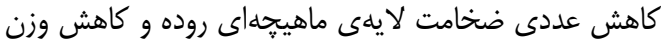

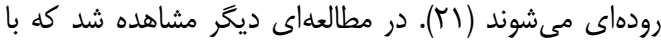

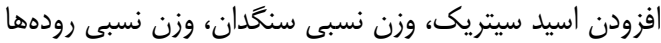
و طول نسبى دئودنوم كاهش يافت كافي كه علت آن آن را مى إتوان در

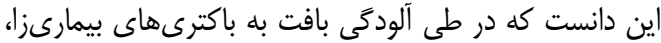

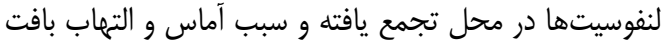

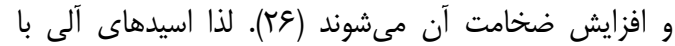

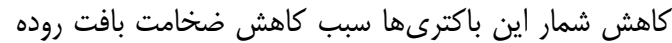

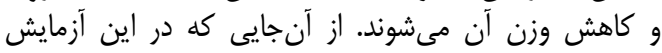

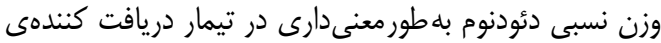

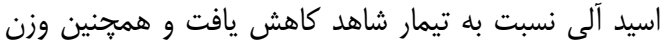

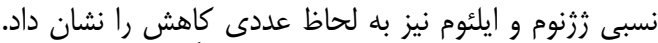

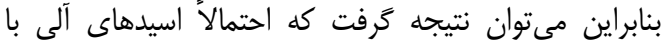

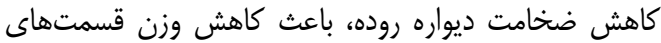

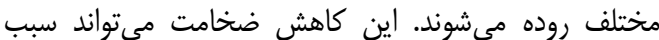

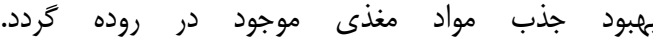

جدول سـ اثر تيمارهاى آزمايشى بر عملكرد جوجههاى گوشتى در دورههاى مختلف آزمايش

Table 3. Effect of experimental treatments on performance of broiler chickens in different period of experiment

\begin{tabular}{|c|c|c|c|c|c|c|c|c|}
\hline $\mathrm{P}$ value & SEM & "رى بيوتيك" & يروبيوتيك & اسيد آلى 'إ' & آنتى بيوتيك' & شاهد & دوره & \\
\hline NS & $10 / 9 .$. & VTY/GN & $V I V / 4$. & $V \cdot V / r I$ & $V \notin q / T \Lambda$ & VGT/QT & $V-r \mid$ & \multirow{3}{*}{ مصرف خوراى (كرم) } \\
\hline NS & GN/DGT & $r$ rqu/. & $r \pi \Delta V / \Delta 1$ & $r r q . / 4 q$ & Tr\&/AI & rYAT/VI & $M T-K T$ & \\
\hline NS & VQ/r IV & $M I r . / W V$ & $r \cdot V \psi / q 1$ & rq৭У/А. & $r \cdot \mid v / \cdot q$ & $\Gamma \cdot F \Delta / \varepsilon \phi$ & V-FT & \\
\hline NS & $11 / r 99$ & FET & $F \mid N / \Delta q$ & FTF/F. & $F T V / \& \&$ & $F A T / A Y$ & $V-r$ & \multirow{3}{*}{ افزايش وزن (كرم) } \\
\hline NS & سبر/אי & $|r \cdot r / v|$ & $1 \pi \Delta \Gamma / r$. & $|r q| / 8$. & IMGT/AF & TMFT/KT & MT-KT & \\
\hline NS & 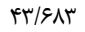 & IVQT/QT & $\mid V K T / \cdot F$ & $|V| g / \cdot 1$ & $|\gamma \wedge \cdot / 9|$ & IVQs/r. & VAtit & \\
\hline NS &.$/ .4 V$ & $1 / 94$ & $1 / \mathrm{NI}$ & $1 / 99$ & $1 / N 1$ & I/GT & $V-r$ & \multirow{3}{*}{ ضريب تبديل غذايى (كرم/ گرم) } \\
\hline NS & \% & $1 / v 9$ & $1 / N \mu$ & $1 / 11$ & $1 / n 1$ & $1 / N \pi$ & MT-KY & \\
\hline NS & . & $1 / N r$ & $1 / N T$ & $1 / 99$ & $1 / n 1$ & $1 / v$. & V-Fit & \\
\hline
\end{tabular}

جدول ع- اثر تيمارهاى آزمايشى بر وزن نسبى قسمتهاى مختلف دستخاه گوارش (gr/kg)

Table 4. Effect of experimental treatments on relative weight of different parts of gastrointestinal tract (gr/kg)

\begin{tabular}{|c|c|c|c|c|c|c|}
\hline سكوم & ايلئوم & رزنوم & د دئودنوم & سنكدان & ييش معده & (2) \\
\hline F/Rg & $I T / V T$ & $\mid r / q T$ & $\gamma / \sim^{\mu} \Lambda^{a}$ & WN/TF & $r / \lambda$. & شاهد \\
\hline r/qr & $11 / . r$ & $\mid r / \Delta r$ & $s / V^{\mathrm{ab}}$ & $N / \Delta$. & $\Delta / 1$. & جيره حاوى آنتىييوتيك' \\
\hline$\mu / \mu$. & $11 / A V$ & $11 / V^{e}$ & $\Delta / / \Delta^{b}$ & $19 / v \Delta$ & $\Delta / \cdot r$ & جيره حاوى اسيد آلى ' \\
\hline $4 / .9$ & 1.149 & $I T / V T$ & $s / r r a b$ & $W / N Q$ & $F / M F$ & جيره حاوى يروبيوتيك" \\
\hline p/rq & $\mid r / 1 \Delta$ & سو/س| & $V / r F^{a}$ & $r \cdot / v^{e}$ & $\Delta / r T$ & جيره حاوى برىييوتيك" \\
\hline | & /MAF &.$/ 981$ &.$/ q q \vee$ & $1 / r v$ & 泟 & SEM \\
\hline ns & $\mathrm{ns}$ & $\mathrm{ns}$ & $*$ & ns & $\mathrm{ns}$ & $P$ value \\
\hline
\end{tabular}


جدول ه- اثر تيمارهاى آزمايش بر طول نسبى قسمتهاى مختلف رودهى باريك (cm/kg)

Table 5. Effect of experimental treatments on relative length of different parts of small intestine $(\mathrm{cm} / \mathrm{kg})$

\begin{tabular}{|c|c|c|c|c|c|}
\hline كولن & سكوم & ايئوم & رزنوم & دئودنوم & تيمار \\
\hline $1 / N f^{0}$ & $V / \& 9$ & $\Gamma \Delta / T \omega^{D}$ & $r r / \Delta q^{\circ}$ & $I F / N$ & شاهد \\
\hline $1 / A F^{D}$ & $N / \Delta \Delta$ & $\Gamma / /{ }^{\mathrm{ad}} \cdot{ }^{\mathrm{a}}$ & $\mathrm{rV} / \mathrm{M}^{\mathrm{ab}}$ & $10 / \% \Delta$ & جيره حاوى آنتى بيوتيك' \\
\hline$r / \kappa r^{a d}$ & $N / N \&$ & $\left(F / /\left.\right|^{a}\right.$ & $\mathrm{rV} / \cdot \mathrm{Q}^{\mathrm{ab}}$ & $I D / V F$ & جيره حاوي \\
\hline$r / 9 \Delta^{\mathrm{a}}$ & N/FT & $f i / v \Delta^{a b}$ & $r N / \Gamma^{a b}$ & $\mid Q / \cdot$. & جيره حاوى - ل جاي \\
\hline$r / 9 q^{a}$ & $9 / .9$ & $F T / Q \mathcal{S}^{\mathrm{a}}$ & $r \cdot / \pi r^{a}$ & $10 / v 9$ & جيره حاوى برى بيوتيك' \\
\hline$\cdot|/ 4|$ &.$/ 499$ & $I / V I V$ & $1 / \mathrm{FVA}^{\prime}$ & D.FT & SEM \\
\hline * & ns & $*$ & $*$ & NS & $P$ value \\
\hline
\end{tabular}

جدول צ- اثر تيمارهاى آزمايشى بر pH قسمتهاى مختلف دستخاه گوارش جوجههاى گوشتى

Table 6. Effect of experimental treatments on pH value of different parts of gastrointestinal tract of broiler chickens

\begin{tabular}{|c|c|c|c|c|c|c|}
\hline ايلئوم & | زئوم & دئودنوم & سنحُدان & ييشمعده & جينهدان & تيمار \\
\hline$\Delta / V \cdot^{a}$ & $\Delta / T v^{a}$ & $r / v r^{\text {alo }}$ & $r / \Lambda r^{a}$ & $r / r r^{a b}$ & $r / 99^{a 0}$ & شاهد \\
\hline$\Delta / \mathrm{r}^{\mathrm{ab}}$ & $\Delta / r^{a}$ & $\Delta / T^{\mathrm{a}}$ & $T / \Delta)^{\text {ad }}$ & $r / q \Delta^{a}$ & $f / r q^{a D}$ & جيره حاوى آنتىبيوتيك' \\
\hline$f / q V^{D}$ & $p / q^{D}$ & $r / 99^{\circ}$ & $r / c^{a b}$ & 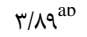 & $\Gamma / \Lambda \Delta^{D}$ & جيره حاوى اسيد آلى 'َ \\
\hline$\Delta / Y \mathcal{G}^{\mathrm{ab}}$ & $r / f \Lambda^{D}$ & $F / \cdot r^{D}$ & r/q سab & $r / v^{a b}$ & $r / g r^{a}$ & جيره حاوى يروبيوتيكى' \\
\hline$\Delta / q^{a}$ & $\Delta / / f^{a b}$ & $f / \mu^{a b}$ & $t / F^{D}$ & $r / r^{D}$ & $f / \& q^{\text {an }}$ & جيره حاوى يرى تيوتيك' \\
\hline • &.$/ M T$ & 每/ & و q & $\cdot / 4 \cdot 9$ &.$/ 19 \wedge$ & SEM \\
\hline$*$ & $*$ & $*$ & $*$ & $*$ & $*$ & $P$ value \\
\hline
\end{tabular}

اين آزمايش مطابق با نظر بسيارى از محققين به طور

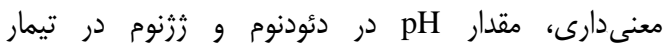

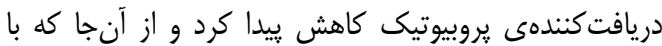

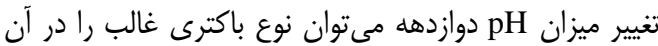

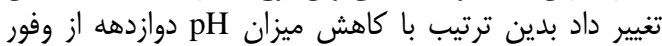

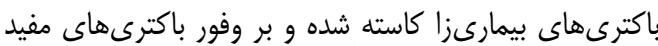

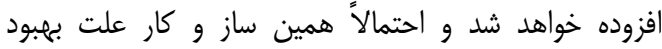

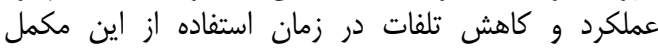

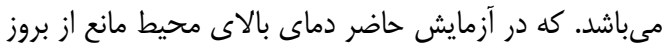
اين اثرات مثبت بر عملكرد كرديد.

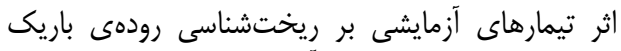

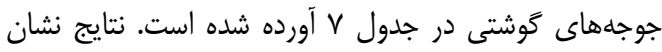

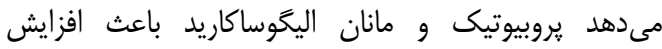

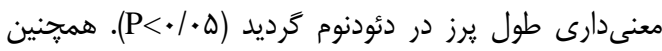

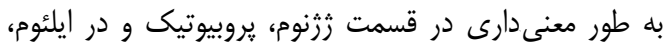

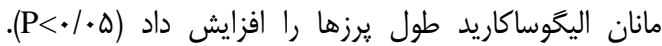

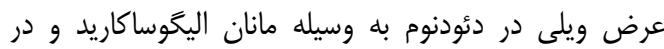

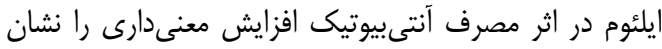

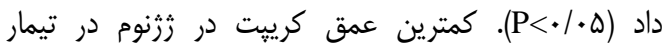

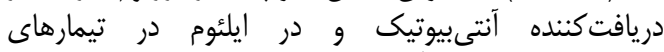

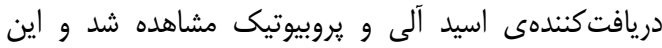

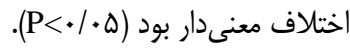

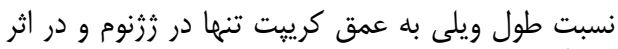

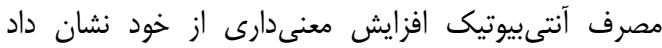

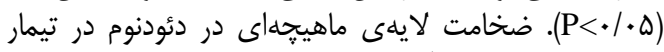

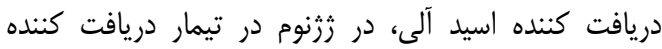

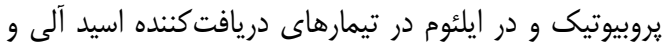

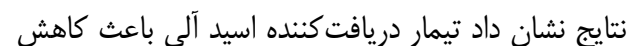

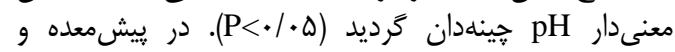

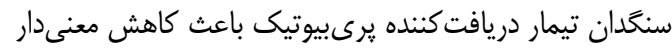
pH

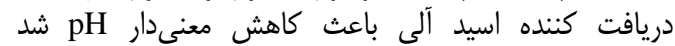

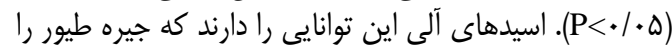

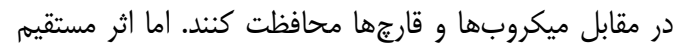

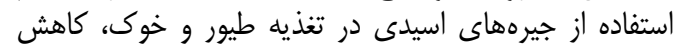
pH

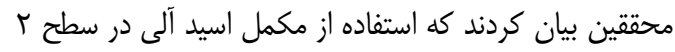
درصد در جيره باعث كاهش معنى دار برار

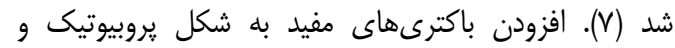

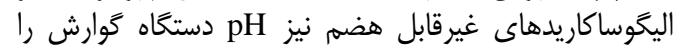
كاهش داده و محيط را براى فعاليت سالمونلا و كلى فيلى باسيلها

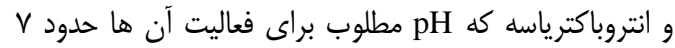

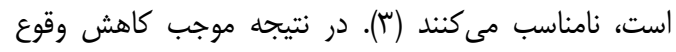

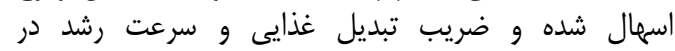

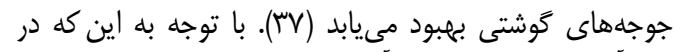

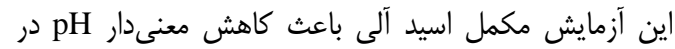

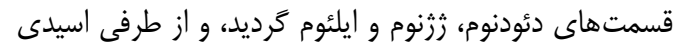

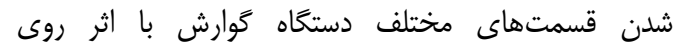

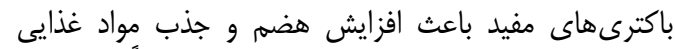

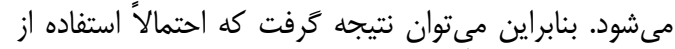

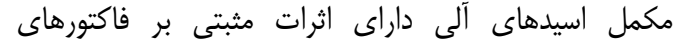

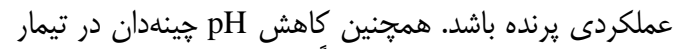

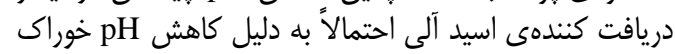

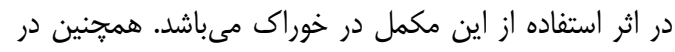




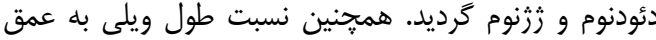

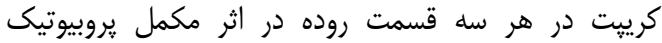
افزايش بيدا كرد كه افزايش اين نسبت ساختار روده را را بيشتر

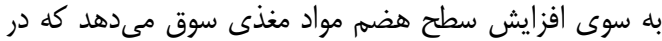

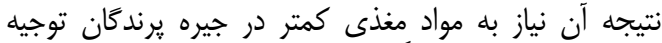

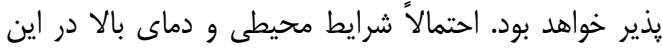

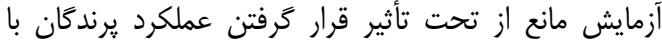

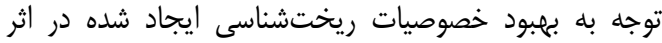

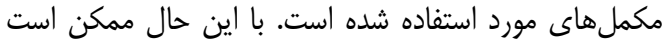

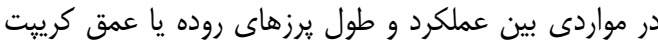

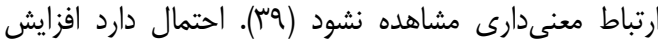

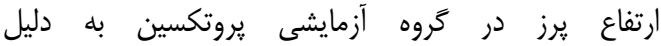

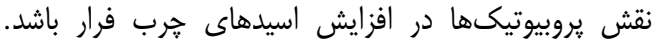

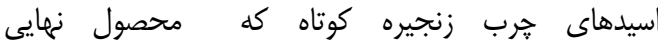

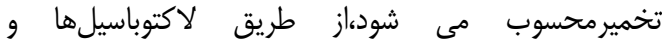

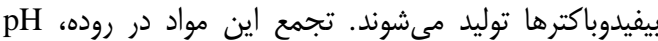

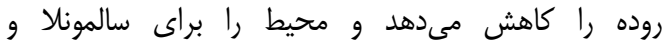

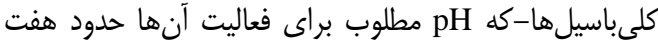

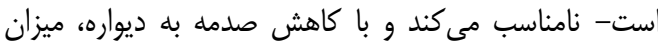

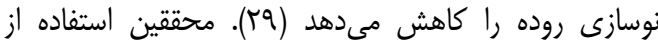

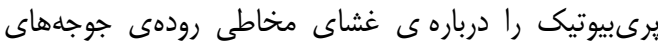

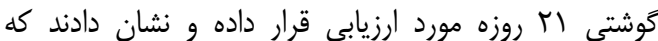

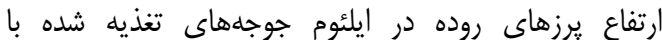

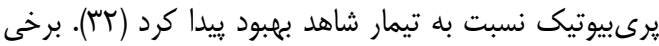

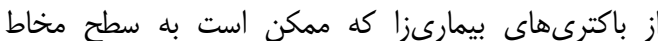

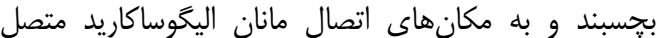

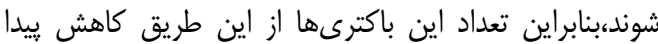

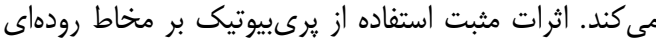

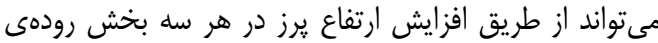

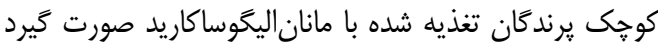

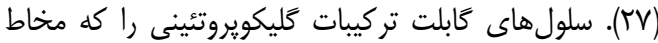

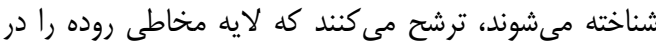

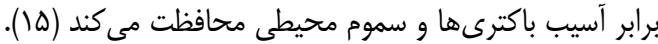

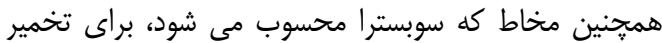

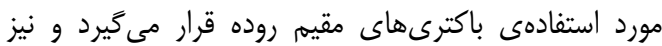

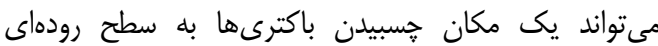

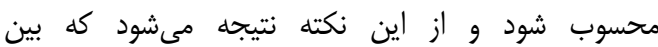

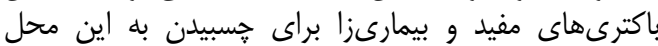

رقابت وجود دارد (9).

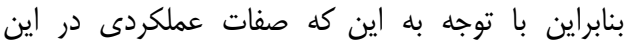

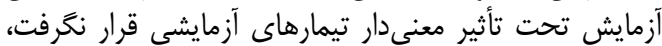

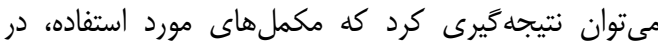

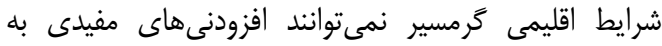

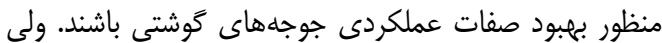

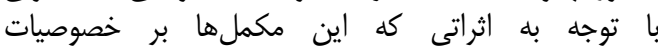

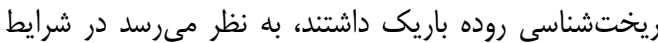

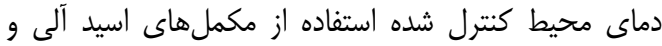

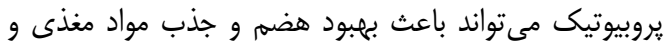
در نتيجه بهببود عملكرد طيور گر دند.

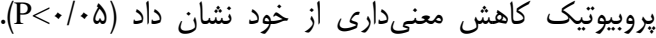

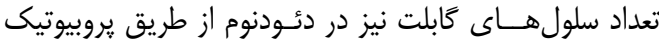

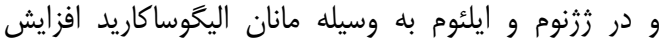

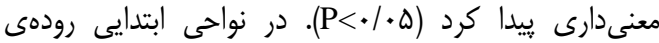

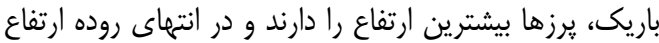

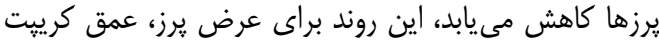

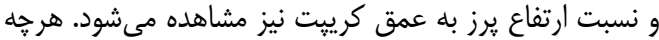

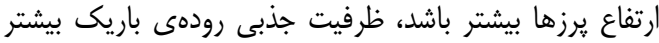

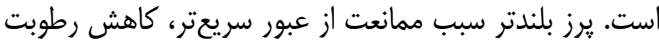

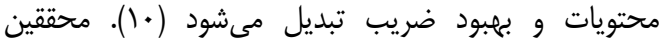

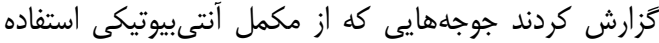

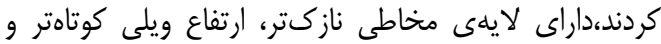

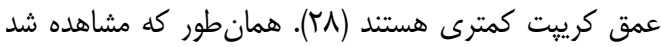

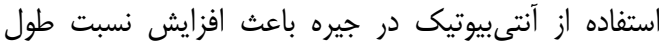

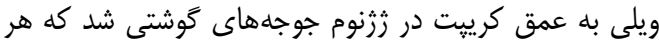

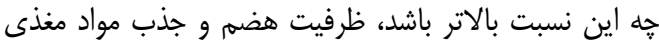

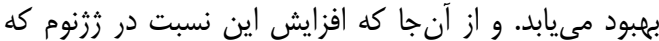

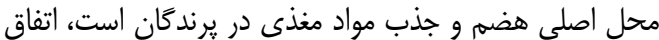

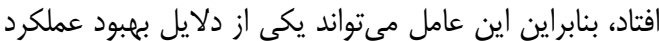

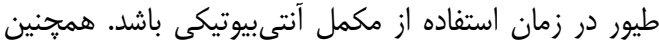

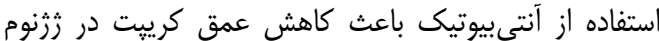

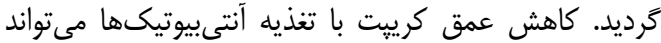

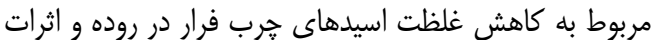

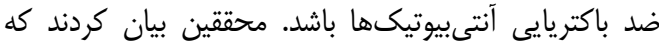

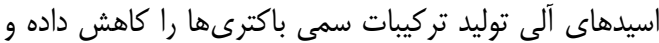

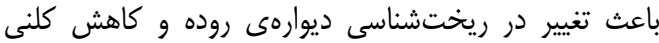

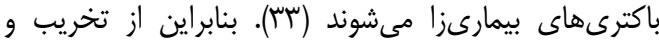

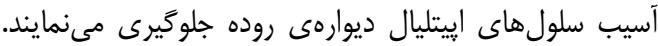

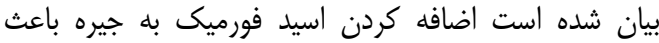

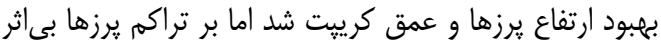

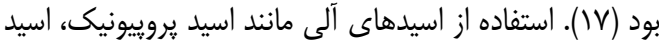

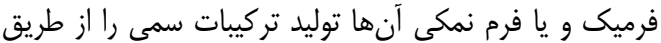

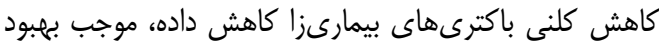

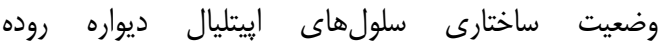

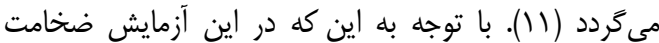

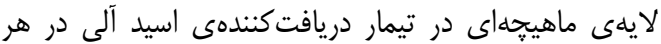

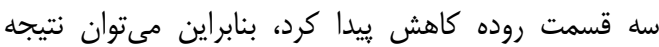

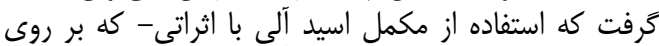

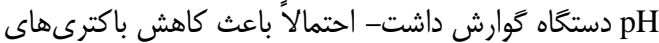

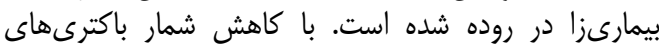

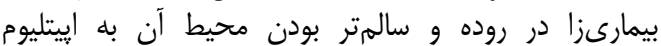

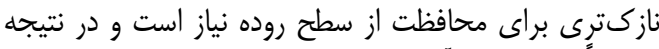

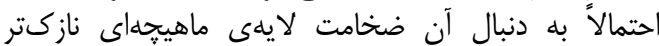

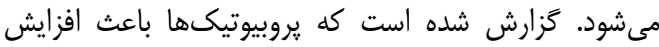

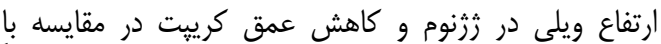

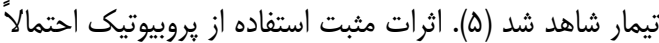

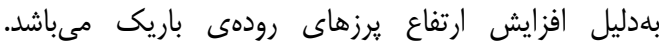

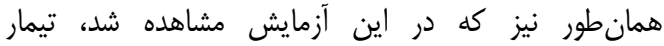
دريافتكنندهى يروبيوتيك باعث افزين ايش طول يرزها در 
جدول V- اثر تيمارهاى آزمايشى بر ريختشناسى رودهى باريك در جوجههاى گوشتى (m) Table 7. Effect of experimental treatments on small intestinal morphology of broiler chicken ( $\mathrm{m}$ )

\begin{tabular}{|c|c|c|c|c|c|c|c|}
\hline تعداد سلولهاى & نهبت طمق كرل يرز & ضاهيجهاه لايه & عمق كرييت & عرض يرز & طول يرز & تيمار & \\
\hline & & & & & & & دئودنوم ( m) \\
\hline (ס/ץ/ & $F / \kappa$. & $r \Lambda \Delta / r^{\mathrm{ad}}$ & $r \pi \Delta / v 1$ & $f \cdot . / \cdot 1^{D}$ & I & شاهد & \\
\hline $9 / 41^{D}$ & $F / 4$ & $r \Psi / r v^{a}$ & $r \cdot r / v r$ & $r A V / V F^{c}$ & $|G T Y / 8|^{D}$ & جيره حاوى آنتى بيوتيك' & \\
\hline $11 / \Delta r^{a b}$ & $r / \widetilde{\mu}$ & $T Y G / T D^{D}$ & एव१/ब & $T M T / \epsilon^{a}$ & $I V \cdot r / q Y^{D}$ & جيره حاوى اسيد آلى' & \\
\hline $10 / 11^{a}$ & $\Delta / \leftarrow \varepsilon$ & $r q \& / V V^{a d}$ & $F \wedge 9 / r F$ & ( & $r \& \Delta T / \cdot r^{\mathrm{a}}$ & جيره حاوى بروبيوتيك' & \\
\hline $\mid r / \Lambda \cdot{ }^{a b}$ & $4 / .9$ & $r \vee \varepsilon / \mathbb{F}^{\mathrm{ab}}$ & $\Delta T \cdot / \Lambda$. & $\Delta r V / c \varphi^{a}$ & $r M I M / \Delta \Lambda^{a}$ & جيره حاوى يرى بيوتيك" & \\
\hline . /qur & $\cdot / \mu \wedge$. & TY/VGG & $r M / / r q$ & $|\varepsilon / \Delta \Psi|$ & $99 / .1$. & SEM & \\
\hline \multirow[t]{2}{*}{$*$} & $\mathrm{~ns}$ & $*$ & ns & $*$ & * & $P$ value & \\
\hline & & & & & & & زثنوم ( m) \\
\hline $\begin{array}{l}\Delta / V D^{D} \\
q / V q^{a D}\end{array}$ & $r / q^{a b}$ & Tr $/ \cdot \Delta^{a}$ & $r q Y / V \Delta^{a d}$ & $r q Y / \cdot r^{\mathrm{ad}}$ & $999 / \mathrm{q}^{\mathrm{D}}$ & شاهد & \\
\hline 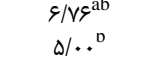 & $r / M v^{a}$ & $r \Delta \cdot / \mathcal{N a b}$ & $r \Psi V / \Lambda e^{D}$ & $T F E / V \Lambda^{D}$ & $1 \cdot r r / I r^{D}$ & جيره حاوى آنتىييوتيك' & \\
\hline $\begin{array}{l}\Delta / . .^{\mathrm{D}} \\
q / r \Delta^{\mathrm{ab}}\end{array}$ & $r / \pi \varepsilon^{\circ}$ & $r \cdot \Delta / q r^{\mathrm{ab}}$ & $P \backslash N / \Lambda)^{a b}$ & $109 / r \Lambda^{c}$ & $111 \Delta / r \Lambda^{a b}$ & جيره حاوى اسيا & \\
\hline $\begin{array}{l}q / T a^{\mathrm{ab}} \\
q / \mathrm{rr}^{\mathrm{a}}\end{array}$ & $r / 99^{a}$ & $T T V / I I^{\circ}$ & $F v \cdot / M \Lambda^{a}$ & 至 & $\mid r^{f} \cdot r / \Lambda r^{a}$ & جيره حاوى يروبيوتيك & \\
\hline $\begin{array}{l}q / r r^{2} \\
. / 9 q 4\end{array}$ & r/q & TrT/ATa & $\kappa r / / \Psi \gamma^{20}$ & $r f \cdot / 19^{\circ}$ & $\mid r F a / r G^{a D}$ & جيره حاوى برى بيوتيك" & \\
\hline \multirow{2}{*}{$*$} & $\cdot / 4 \cdot 9$ & $r \cdot / V I I$ & $r \Delta / T V V^{\epsilon}$ & $1 . / 94 \mathrm{~F}$ & $V \cdot / V \Delta \Lambda$ & SEM & \\
\hline & * & * & * & * & * & $P$ value & \\
\hline$F / \mu)^{c}$ & $f / r$. & 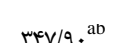 & $r m \mathcal{E} \mid \mathbb{E}^{\mathrm{D}}$ & rew/N $\widetilde{\alpha}^{\mathrm{ab}}$ & $V(\Delta) / \Delta Y^{D}$ & to & ايلئوم ( m) \\
\hline$V / q^{a}{ }^{a}$ & $r / . r$ & 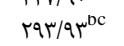 & $r \digamma q / T v^{a D}$ & $r \varepsilon V / V F^{a}$ & $M M / q .{ }^{D}$ & جيره حاوى آنتي ييوتيك' & \\
\hline$\Delta / .{ }^{c a}$ & $F / r q$ & $r \Delta \cdot / \Lambda .^{c a}$ & $r \cdot V / r \Lambda^{D}$ & $19 / / 9 .^{\circ}$ & $V K r / Y I^{D}$ & جيره حاوى اسيد آلى & \\
\hline$g / T I^{D}$ & e/rq & $r|l / r|^{\mathrm{a}}$ & $r \cdot 9 / 4 \mathrm{H}^{\mathrm{D}}$ & $r \in g / \Lambda r^{a b}$ & $W W / .9^{D}$ & جيره حاوى بروبيوتيك' & \\
\hline$N(9)^{a}$ & $\% / \cdot \Delta$ & $r v e / r q^{a}$ & $r . / / f^{a}$ & $\Gamma \leftarrow \Gamma / \backslash \mathcal{F}^{\mathrm{aD}}$ & $|1 F| / r q^{a}$ & جيره حاوى برى بيوتيك' & \\
\hline | (1\%/. (1) & $\cdot / 494$ & $\mid \varepsilon / \% \cdot 1$ & $\mid r / \Lambda \cdot r$ & $|F / r q|$ & $F F / 109$ & SEM & \\
\hline * & ns & * & * & * & * & $P$ value & \\
\hline \multicolumn{8}{|c|}{ 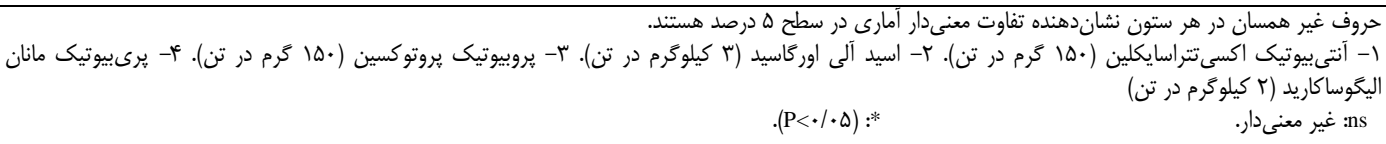 } \\
\hline
\end{tabular}

1. Acikgoz, Z., H. Bayraktar and O. Altan. 2011. Effects of Formic acid administration in the drinking Water on Performance, Intestinal Microflora and Carcass Contamination in Male Broilers under High Ambient Temperature. Asian Austrian Journal of Animal Science, 24: 96-102.

2. Al-Natour, M.Q. and K.M. Alshawabkeh. 2005. Using varying levels of formic acid to limit growth of Salmonella gallinarum in contaminated broiler feed. Asian-Austal Journal of Animal Science, 18: 390-395.

3. Angel, R., R.A. Dalloul and J. Doerr. 2005. Performance of broiler chickens fed diets supplemented with a Direct-Fed Microbial. Poultry Science, 84: 1222-1231.

4. Bonnet, S., P.A. Geraert, M. Lessire, B. Carre and S. Guillaumin. 1997. Effect of high ambient temperature on feed digestibility in broilers. Poultry Science, 76: 857-863.

5. Chichlowisk, M.J., B.W. Croom, L. McBride, G. Daniel, R. Davis and M.D. Koci. 2007. Direct-fed microbial primalac and salinomycin modulate whole-body and Intestinal oxygen consumption and Intestinal Mucosal cytokine production in the broiler chick. Poultry Science, 86: 1100-1106.

6. Clements, M.L., M.M. Levine and R.E. Black. 1981. Lactobacillus prophylaxis for diarrhea due to entrotoxigenic escherchia coli. Antimicrob Agents and Chemotherapy, 20: 104-108.

7. Clik, K. and I. Ersoy. 2003. The using of organic acid in California turkey chicks and its effects on performance before pasturing. Poultry Science, 2: 446-448.

8. Coates, M.E., R. Fuller, G.F. Harrison, M. Lev and S.F. Suffolk. 1963. A comparison of the growth of chicks in the Gustafsson germ-free apparatus and in a conventional environment, with and without dietary supplements of penicillin. British Journal of Nutrition, 17: 141-150.

9. Craven, S.E. and D.D. Williams. 1998. In vitro attachment of Salmonella Ttyphimurium to chicken cecal mucus: Effect of cations and pretreatment with Lactobacillus spp. isolated from the intestinal tracts of chickens. Journal of Food Protection, 61: 265-271.

10. Deschepper, K., M. Lippens, G. Huyghebaert and K. Molly. 2003. The effect of aromabiotic and GALI D'OR on technical performances and intestinal morphology of broilers. In: Proccedings of $14^{\text {th }}$. European Symposium on poultry nutrition. August. Lillehammer, Norway. 189.

11. Dibner, J.J. and P. Buttin. 2002. Use of organic acids as a model to study the impact of gut microflora on nutrition and metabolism. Journal of Applied Poultry Research, 11: 453-463.

12. Eidelsburger, U. 1998. Feeding short-chain organic acids to pigs. In: Recent Advances in Animal Nutrition, Garnsworthy, P.C. and Wiseman, J. (Eds), (pp: 93-106). Nottingham University press, Nottingham.

13. Esteva-Garcia, E. and S. Mack. 2000. The effect of DL-methionine and betaine on growth performance and carcass characteristics in broilers. Animal Feed Science and Technology, 87: 151159. 
14. Farhoomand, P. and A. Dadvend. 2007. Carcass weight, growth performance and intestinal organs size of broilers fed graded levels of Saccharomycese cervisiae supplementation diets. Pakistan Journal of Biological Science, 10: 1870-1874.

15. Forstner, J.T. 1978. Intestinal mucins in health and disease. Digestion, 17: 234-263.

16. Fuller, R. 1989. A review: Probiotics in man and animals. Journal of Applied Bacteriology, 66: 365378.

17. Garcia, V., P. Catala-Gregori, F. Hernandez, M.D. Megıas and J. Madrid. 2007. Effect of formic acid and plant extracts on growth, nutrient digestibility, intestine mucosa morphology, and meat yield of broilers. Journal of Applied Poultry Research, 16: 555-562.

18. Garrido, N.M., M. Skjveheim, H. Oppegaard and H. Sorum. 2004. Acidified litter benefits the intestinal flora balance of broiler chickens. Applied and Environmental microbiology, 70: 5208-5213.

19. Gibson, G.R. and M.B. Roberfroid. 1995. Dietary modulation of the colonic microbiotica: introducing the concept of probiotics. Journal of Nutrition, 125: 1401-1412.

20. Goldin, B.R. 1998. Health benefits of probiotics. British Journal of Nutrition, 80: 203-207.

21. Gunal, M., G. Yayli, O. Kaya, N. Karahan and O. Sulak. 2006. The effects of antibiotic growth promoter, probiotic or organic acid supplementation on performance, intestinal microflora and tissue of broilers. International Journal of Poultry Science, 5: 149-155.

22. Gunes, H., H. Cerit and A. Altinel. 2001. Effect of organic acid, probiotic and antibiotic on performance and carcass yield of broilers. Sociedade Brasileira de Zootecnia, 302-308.

23. Hampson, D.J. 1986. Alteration in piglet small intestinal structure at weaning. Research in Veterinary Science, 40: 39-40.

24. Izat, A.L., N.M. Tidwell, R.A. Thomas, M.A. Reiber, M.H. Adams, M. Colberg and P.W. waldroup. 1990. Effects of a buffered propionic acid in diets on the performance of broiler chickens and on microflora of the intestine and carcass. Poultry Science, 69: 818-826.

25. Kafilzadeh, F. and M.R. Safariparvar. 2002. Effect of different levels of Probiotic IMONOBAC on performance of broiler. Journal of Agriculture Science and Natural Research, 4: 173-179.

26. Kaya, C.A. and S.D. Tuncer. 2009. The effects of an organic acids and etheric oilsmixture on fatting performance, carcass quality and some blood parameters of broilers. Journal of Animal and Veterinary Advances, 8: 94-98.

27. Macari, M. and A. Maiorka. 2000. Funçao gastrintestinal e seu impacto no rendimento avícola. In: anais da conferência apinco de ciencia e tecnologia avícolas. Campinas: FACTA, 2: 161-174.

28. Miles, R.D., G.D. Butcher, P.R. Henry and R.C. Littell. 2006. Effect of antibiotic growth promoters on broiler performance, intestinal growth parameters and qualitative morphology. Poultry Science, 85 : 476-85.

29. Mohan, K.O.R. and C.K. James. 1988. The role of Lactobacillus sporogens (probiotic) as feed additives. Poultry Guide, 25: 37-39.

30. Mountzouris, K., H. Beneas, P. Tsirtsikos, E. Kalamara and K. Fegeros. 2006. Evaluation of the effect of a new probiotic product on broiler performance and cecal microflora composition and metabolic activities. International poultry scientific forum atlanta, Georgia January, 23-24.

31. Pardue, S.L. and J.P. Thaxton. 1986. Ascorbic acid in poultry. a review. World's poultry. Science, 25: 42: 107.

32. Pelicano, E.R.L., P.A. Souza, H.B.A. Souza, D.F. Figueiredo, M.M. Boiago, S.R. Carvalho and V.F. Bordon. 2005. Intestinal mucosa development in broiler chickens fed natural growth promoters. Brazilian Journal of Poultry Science, 7: 221-229.

33. Pourhasan, H., Sh. Rahimi, M.A. Karimi Torshiz and T. Zahraei Salehi. 2008. Effect of organic acids on intestinal microflora and morphology of broiler chicks. Journal of Veterinary Research, 63: 283290.

34. Radcliffe, J. 2000. British supermarkets: forging changes in poultry nutrition. Austral. Poultry Science. Sympos, 12: 25-31.

35. Sanders, M. 1999. Probiotics. Food Technology, 53: 67-77.

36. SAS Institute. 2004. User,s Guids Version 9.1: Statistics. SAS Institute, Cary N.C.

37. Schneitz, C., T. Kiskinen, V. Toivonen and M. Nasi. 1998. Effect of BROILAC on the physiochemical conditions and nutrient digestibility in the gastrointestinal tract of broilers. Poultry Science, 77: 426-432.

38. Sell, J.L., C.R. Angel, F.J. Piquer, E.G. Mallarino and H.A. Al-Batshan. 1991. Development patterns of selected characteristics of the gastrointestinal tract of young turkey. Poultry Science, 70: 12001205.

39. Vieira, S.L., O.A. Oyarzabal, D.M. Freitas, J. Berres, J.E.M. Pena, C.A. Torres and J.L.B. Coneglian. 2008. Performance of broilers fed diets supplemented with sanguinarine-like alkaloids and organic acids. Journal of Applied Poultry Research, 17: 128-133.

40. Waldroup, A. and W. Kanis. 1995. Performance characteristics and microbiological aspects of broiler fed diets supplemented with organic acids. Journal of Food Protection, 58: 482-489.

41. Yang, Y., P.A. Iji and M. Choct. 2009. Dietary modulation of gut microflora in broiler chickens: a review of the role of six kinds of alternatives to in-feed antibiotics. World's Poultry Science, 65: 97114.

42. Yusrisal, Y. and T. Chen. 2003. Effect of adding chicory fructans in feed on broiler growth performance, serum cholesterol and intestinal length. International Journal of Poultry Science, 2: 214219. 


\title{
Evaluation of Production Performance and Gut Morphology of Broiler Chickens Fed with Antibiotic, Organic Acid, Probiotic and Prebiotic in Tropical Conditions
}

\author{
Mosayeb Shalaei $^{1}$, Seyyed Mohammad Hosseini ${ }^{2}$ and Nazar Afzali ${ }^{3}$ \\ 1- M.Sc., University of Birjand (Corresponding Author: Mosayeb_shalaey @ yahoo.com) \\ 2 and 3- Assistant Professor and Professor, University of Birjand \\ Received: November 19,2013 Accepted: April 6, 2014
}

\begin{abstract}
The experiment was conducted to evaluate the effect of antibiotic, organic acid, probiotic and prebiotic on performance and small intestinal morphology in male Ross 308 broiler chicks for 42 days in tropical conditions. The study was completely randomized design with 5 treatments, 4 replicates and 8 chicks per each. The treatments were: $\mathrm{T}_{1}$ : basal diet, $\mathrm{T}_{2}: \operatorname{diet}$ containing antibiotic oxytetracycline $\left(150 \mathrm{~g} /\right.$ ton of feed), $\mathrm{T}_{3}$ : diet containing organic acid orgacid $\left(3 \mathrm{~kg} /\right.$ ton of feed), $\mathrm{T}_{4}$ : diet containing probiotic protoxin $\left(150 \mathrm{~g} /\right.$ ton of feed) and $\mathrm{T}_{5}$ : diet containing prebiotic mannan oligosaccharide $(2 \mathrm{~kg} / \mathrm{ton}$ of feed $)$. The results showed that the experimental diets had no significant effect on feed consumption, live weight and feed conversion ratio. The relative length of jejunum and ileum by prebiotic significantly increased $(\mathrm{P}<0.05)$. The acidity of different parts of the small intestine, showed significantly decreased by consumption of organic acid supplementation $(\mathrm{P}<0.05)$. The treatment receiving probiotic significantly increased the height of the villi in duodenum and jejunum $(\mathrm{P}<0.05)$. Also treatment receiving antibiotic and probiotic significantly increased villi height to crypt depth ratio in the jejunum $(\mathrm{P}<0.05)$. The thickness of the muscle layer in jejunum and ileum by consumption of organic acid and probiotic significantly decreased $(\mathrm{P}<0.05)$. Generally, the results of the present study showed the positive effects of supplements used on the morphological characteristics of small intestine, but did not have any difference in the performance of broiler chickens.
\end{abstract}

Keywords: Broiler chickens, Dietary supplements, Intestinal morphology, Performance 